\title{
Quantization of Magnetic Poisson Structures
}

\author{
LMS/EPSRC Durham Symposium on Higher Structures in M-Theory
}

Richard J. Szabo ${ }^{a, *}$

\begin{abstract}
We describe three perspectives on higher quantization, using the example of magnetic Poisson structures which embody recent discussions of nonassociativity in quantum mechanics with magnetic monopoles and string theory with non-geometric fluxes. We survey approaches based on deformation quantization of twisted Poisson structures, symplectic realization of almost symplectic structures, and geometric quantization using 2-Hilbert spaces of sections of suitable bundle gerbes. We compare and contrast these perspectives, describing their advantages and shortcomings in each case, and mention many open avenues for investigation.
\end{abstract}

\section{Introduction}

In this contribution we will study three perspectives on the problem of higher quantization, which at present is a problem not understood to the level of ordinary quantization schemes such as geometric quantization or, more concretely, canonical quantization in quantum mechanics. We do not attempt a general discussion of the problem; see e.g. [1-3] for detailed overviews of the issues surrounding higher quantization generically. Instead, we focus on a particularly simple and tractable class of models of physical significance, that we call 'magnetic Poisson structures', in which precise statements and advancements can be made from both mathematical and physical perspectives. These are special examples of nondegenerate twisted Poisson, or equivalently almost symplectic, structures which are related to recent discussions of nonassociativity in quantum mechanics and in nongeometric string theory.

We define and motivate the relevant structures in Section 2, and describe the general quantization problem involved. We then offer three perspectives on how to tackle this quantization problem, starting with the most concrete framework and ending with the most abstract one. Section 3 reviews the well-known approach through deformation quantization. In Section 4 we describe a new ap- proach through an extension of the well-known procedure of symplectic realization in Poisson geometry to the case of twisted (or more generally quasi-) Poisson structures. Finally, in Section 5 we describe an approach based on a higher version of geometric quantization which brings the formalism of higher structures into full play by regarding 'higher quantum states' as sections of a suitable bundle gerbe, the appropriate higher analog of the line bundles usually employed in ordinary geometric quantization. Each of these approaches has their own advantages, but also several deficiencies which we explain in detail in the following.

\section{Magnetic Poisson structures}

We begin by defining and motivating the specific twisted Poisson structures that we will attempt to quantize. We shall then describe the specifics of the quantization problem we wish to address.

\subsection{Definition}

We work in the simple setting of the $d$-dimensional vector space $M=\mathbb{R}^{d}$, which we will refer to as 'configuration space' in the following; local coordinates on $M$ are denoted $x$. We write $M^{*}$ for the dual vector space and call it 'momentum space', with local coordinates $p$. The (trivial) cotangent bundle $\mathcal{M}=T^{*} M=M \times M^{*}$ is called 'phase space'; it has local coordinates $X=(x, p)$ and the canonical symplectic form

$\sigma_{0}\left(X, X^{\prime}\right)=p \cdot x^{\prime}-p^{\prime} \cdot x$

\footnotetext{
* Corresponding author e-mail: R.J.Szabo@hw.ac.uk

a Department of Mathematics, Heriot-Watt University, Edinburgh EH14 4AS, United Kingdom; Maxwell Institute for Mathematical Sciences, Edinburgh, United Kingdom; The Higgs Centre for Theoretical Physics, Edinburgh, United Kingdom
} 
where a dot denotes the canonical duality pairing between vectors and covectors.

We fix a (not necessarily closed) two-form $\rho \in \Omega^{2}(M)$ on configuration space $M \subset \mathscr{M}$ and call it a 'magnetic field', for reasons that will become clear from the applications we describe below. It deforms the symplectic structure $\sigma_{0}$ to an almost symplectic form

$\sigma_{\rho}=\sigma_{0}-\rho$,

which is always non-degenerate (because $\sigma_{0}$ is) but is closed if and only if $\rho$ is closed; in (2) we of course mean the pullback of $\rho$ under the cotangent bundle projection $\mathscr{M} \longrightarrow M$, and we shall frequently abuse notation in this way since all considerations in the following occur in this simple topologically trivial setting. Its inverse $\theta_{\rho}=\sigma_{\rho}^{-1}$ gives a bivector which defines the magnetic Poisson algebra on $C^{\infty}(\mathscr{M})$ with brackets

$$
\{f, g\}_{\rho}=\theta_{\rho}(\mathrm{d} f \wedge \mathrm{d} g)
$$

for smooth complex-valued phase space functions $f, g \in$ $C^{\infty}(\mathscr{M})$. In particular, for the coordinate functions $x^{i}(X)=$ $x^{i}$ and $p_{i}(X)=p_{i}$, with $i=1, \ldots, d$, one has

$$
\begin{aligned}
& \left\{x^{i}, x^{j}\right\}_{\rho}=0, \\
& \left\{x^{i}, p_{j}\right\}_{\rho}=\delta^{i}{ }_{j}, \\
& \left\{p_{i}, p_{j}\right\}_{\rho}=-\rho_{i j}(x),
\end{aligned}
$$

where $\rho=\frac{1}{2} \rho_{i j}(x) \mathrm{d} x^{i} \wedge \mathrm{d} x^{j}$ (with implicit summation over repeated upper and lower indices always understood).

These brackets do not generally define a Poisson algebra but rather an $H$-twisted Poisson structure on $\mathscr{M}$, with twisting given by the three-form $H=\mathrm{d} \rho$ on $M$ that we shall call a 'magnetic charge'. This means that the Schouten bracket of the bivector $\theta_{\rho}$ with itself, which governs the associativity of the brackets defined by (3), is given by the trivector

$$
\left[\theta_{\rho}, \theta_{\rho}\right]_{S}=\bigwedge^{3} \theta_{\rho}^{\sharp}\left(\mathrm{d} \sigma_{\rho}\right),
$$

where $\theta_{\rho}^{\sharp}$ denotes the natural contraction of forms to vectors by the non-degenerate bivector $\theta_{\rho}$. It vanishes if and only if $H=0$, while it generically gives a nonassociative algebra with Jacobiators

$$
\begin{aligned}
\{f, g, h\}_{\rho} & :=\frac{1}{3}\left(\left\{f,\{g, h\}_{\rho}\right\}_{\rho}-\left\{\{f, g\}_{\rho}, h\right\}_{\rho}-\left\{g,\{f, h\}_{\rho}\right\}_{\rho}\right) \\
& =\left[\theta_{\rho}, \theta_{\rho}\right]_{S}(\mathrm{~d} f \wedge \mathrm{d} g \wedge \mathrm{d} h) .
\end{aligned}
$$

In particular, on coordinate functions the only nonvanishing Jacobiators are given by

$\left\{p_{i}, p_{j}, p_{k}\right\}_{\rho}=-H_{i j k}(x)$,

where $H=\frac{1}{3 !} H_{i j k}(x) \mathrm{d} x^{i} \wedge \mathrm{d} x^{j} \wedge \mathrm{d} x^{k}$. This is a generalization of the nonassociative algebras that were introduced in the physics literature of the 1980's in the context of the Günaydin-Zumino model [4], and they are a natural playground for higher structures as we explain in the following.

There are obvious generalizations of this model that one can envisage. One can of course replace $M$ with any manifold (not necessarily a vector space). The cotangent bundle $\mathscr{M}=T^{*} M$ can likewise be replaced by any Lie algebroid $\mathscr{L}$ over $M$, with $\rho \in \Gamma\left(M, \wedge^{2} \mathscr{L}^{*}\right)$ a Lie algebroid two-cochain which defines a central extension of $\mathscr{L}$. Then $H=\mathrm{d} \rho$ defines a Lie algebroid three-cocycle with values in the kernel of the anchor map of the extension and hence can be used to endow $\mathscr{L}$ with the structure of an $H$-twisted Lie algebroid [5], which is in particular a 2-term $L_{\infty}$-algebroid. However, here we stick to this concrete and simple example as it will capture the essential features that we wish to describe in this contribution. Let us first briefly explain two motivations from physics for being interested in the quantization of such structures.

\subsection{Application I: magnetic monopoles}

Our first application is to quantum mechanics. The magnetic Poisson structure in $d=3$ dimensions with $\rho_{i j}=$ $e \varepsilon_{i j k} B^{k}$ governs the motion of an electric charge $e$ in a magnetic field $\mathbf{B}$ on $\mathbb{R}^{3}$. When the two-form $\rho$ is closed, $\mathrm{d} \rho=0$, this corresponds to the classical Maxwell theory without magnetic sources, $\operatorname{div} \mathbf{B}=0$, and with a globally defined magnetic vector potential $\mathbf{A}$ on $\mathbb{R}^{3}$ such that $\mathbf{B}=\operatorname{curl} \mathbf{A}$. This is the case in which the magnetic Poisson structure yields an associative Poisson algebra.

The simplest instance of nonassociativity comes from Dirac's modification of Maxwell's theory, which considers a singular delta-function distributed source of magnetic charge at the origin $\mathbf{0}$ of $\mathbb{R}^{3}$ representing a Dirac monopole. The magnetic field sourced by the Dirac monopole on $\mathbb{R}^{3} \backslash\{\mathbf{0}\}$ is given by

$\mathbf{B}_{\mathrm{D}}=g \frac{\mathbf{x}}{|\mathbf{x}|^{3}}=\operatorname{curl} \mathbf{A}_{\mathrm{D}}$,

where $g$ is the magnetic charge and the local magnetic vector potential

$\mathbf{A}_{\mathrm{D}}=\frac{g}{|\mathbf{x}|} \frac{\mathbf{x} \times \mathbf{n}}{|\mathbf{x}|-\mathbf{x} \cdot \mathbf{n}}$ 
has an additional Dirac string singularity along the semiinfinite line emanating from the location of the monopole at $\mathbf{0}$ in the direction of a fixed unit vector $\mathbf{n}$. This makes a modest connection between higher structures and experiment, in that analog systems of Dirac monopoles have been observed through neutron scattering events off of spin ice pyrochlore lattices, see e.g. [6, 7] for early reports. These lattices have tetrahedral atomic arrangements with magnetic dipoles through the corners of the tetrahedra, and local magnetic pole defects in the lattice can be observed in interference patterns from interactions with neutrons, which themselves have a dipole moment, and an external magnetic field. In this sense Dirac strings and monopoles arise as emergent states of matter; see [8] for a more detailed discussion in the present context and further references.

In this contribution we are ultimately interested in the cases where the twisting three-form $H=\mathrm{d} \rho \neq 0$ is non-singular and describes smooth distributions of magnetic charge. Although not yet visible in experiment, one can regard such distributions as arising in an effective framework where we treat a system of Dirac monopoles in a long wavelength limit at scales much larger than the lattice spacing used in realistic scenarios. In such a setting one must account for nonassociativity along smooth submanifolds, and not just at the point supports of Dirac monopoles. Then a foundational question about the quantum dynamics of electric charge in such distributions arises:

- What is a sensible framework for nonassociative quantum mechanics?

By 'sensible' we mean a formalism that agrees with the usual physical requirements of a quantum theory and which has the potential to be experimentally tested.

\subsection{Application II: locally non-geometric fluxes}

A somewhat more speculative application is to string theory. Consider the magnetic duality transformation $(x, p) \longmapsto(p,-x)$ on the phase space $\mathscr{M}$ (for any $d$ ), which preserves the canonical symplectic structure $\sigma_{0}$. In this case we can trade the configuration space two-form $\rho \in$ $\Omega^{2}(M)$ with a momentum space two-form $\beta \in \Omega^{2}\left(M^{*}\right)$ such that the twisted Poisson brackets among coordinate functions become

$$
\begin{aligned}
& \left\{x^{i}, x^{j}\right\}_{\beta}=-\beta^{i j}(p), \\
& \left\{x^{i}, p_{j}\right\}_{\beta}=\delta^{i}{ }_{j}, \\
& \left\{p_{i}, p_{j}\right\}_{\beta}=0,
\end{aligned}
$$

where $\beta=\frac{1}{2} \beta^{i j}(p) \mathrm{d} p_{i} \wedge \mathrm{d} p_{j}$. In this case the twisting three-form is the ' $R$-flux' $R=\mathrm{d} \beta \in \Omega^{3}\left(M^{*}\right)$ which describes a nonassociative configuration space with the nonvanishing Jacobiator among coordinate functions given by

$\left\{x^{i}, x^{j}, x^{k}\right\}_{\beta}=-R^{i j k}(p)$,

where $R=\frac{1}{3 !} R^{i j k}(p) \mathrm{d} p_{i} \wedge \mathrm{d} p_{j} \wedge \mathrm{d} p_{k}$. This dynamical system is called the R-flux model, and it conjecturally describes the phase space of closed strings propagating in 'locally non-geometric' $R$-flux backgrounds, see e.g. [9-13]. In this example another foundational question arises:

i) What substitutes for canonical quantization of locally non-geometric closed strings?

\subsection{Magnetic translation operators}

Let us now describe the quantization problem for magnetic Poisson structures in some generality. Quantization should be a linear map $f \longmapsto \mathscr{O}_{f}$ from functions $f \in C^{\infty}(\mathscr{M})$ to a collection of symbols $\mathscr{O}_{f}$ which close under some $\mathbb{C}$-linear operation transporting the pointwise multiplication of functions in a suitable sense; we leave the precise specification of these operators intentionally vague for the moment, as their definition will depend on both the details of the magnetic Poisson structure and on the specific quantization scheme that we adopt. The minimal requirement is that they should reproduce the classical brackets (3) at leading order in a deformation parameter $\hbar$, which in physical scenarios we would like to identify with Planck's constant of quantum mechanics. In other words, we demand that the corresponding commutator brackets of the symbols satisfy

$\left[\mathscr{O}_{f}, \mathscr{O}_{g}\right]=\mathrm{i} \hbar \mathscr{O}_{\{f, g\}_{\rho}}+O\left(\hbar^{2}\right)$,

which just mimicks the correspondence principle of quantum mechanics. Note that the quantization map is only required to be a homomorphism of the bracket algebras to leading order in $\hbar$; even in the simplest physical examples of canonical quantization, the homomorphism property is violated in general at order $\hbar^{2}$. An exception is the restriction of the map to linear (or quadratic) polynomials in the coordinate functions of $C^{\infty}(\mathscr{M})$, which preserves the fundamental brackets (4):

$\left[\mathscr{O}_{x^{i}}, \mathscr{O}_{x^{j}}\right]=0$,

$\left[\mathscr{O}_{x^{i}}, \mathscr{O}_{p_{j}}\right]=\mathrm{i} \hbar \delta^{i}{ }_{j} 1_{\mathscr{O}}$,

$\left[\mathscr{O}_{p_{i}}, \mathscr{O}_{p_{j}}\right]=-\mathrm{i} \hbar \rho_{i j}\left(\mathscr{O}_{x}\right)$, 
where $1_{\mathscr{O}}=\mathscr{O}_{1}$ is the image of the constant unit function 1 on $\mathscr{M}$ under the quantization map. This generally defines a nonassociative algebra whose non-vanishing Jacobiators are given by

$\left[\mathscr{O}_{p_{i}}, \mathscr{O}_{p_{j}}, \mathscr{O}_{p_{k}}\right]=-\hbar^{2} H_{i j k}\left(\mathscr{O}_{x}\right) 1_{\mathscr{O}}$.

The symbols $\rho_{i j}\left(\mathscr{O}_{x}\right)$ and $H_{i j k}\left(\mathscr{O}_{x}\right)$ depend in general on a choice of ordering, which is ambiguous; even in the simplest examples of standard canonical quantization, different ordering prescriptions lead to different quantization maps.

Whatever the quantization scheme, a fundamental ingredient in the quantization of magnetic Poisson structures consists of the magnetic translation operators

$\mathscr{P}_{v}=\exp \left(\frac{\mathrm{i}}{\hbar} \mathscr{O}_{p \cdot v}\right)$,

defined symbolically here as global symbols via formal power series expansions for fixed configuration space vectors $v=\left(\nu^{i}\right) \in M=\mathbb{R}^{d}$. Their significance is that their conjugation action on the symbols $\mathscr{O}_{f}$ implements an action of the translation group $T=\mathbb{R}^{d}$; indeed, a formal implementation of the first two commutation relations in (13) yields

$\mathscr{P}_{\nu}^{-1} \mathscr{O}_{x^{i}} \mathscr{P}_{\nu}=\mathscr{O}_{x^{i}+v^{i}}$

on the basic symbols corresponding to the configuration space coordinates. This is expected on physical grounds from the example of a charged particle in a magnetic field considered in Section 2.2: A non-zero background magnetic field breaks the exact translational symmetry of the classical dynamical system, but a remnant of this symmetry remains after quantization in the form of a "projective" representation of the translation group $T=\mathbb{R}^{d}$, in a suitable sense that we will make precise in the following. In ordinary quantum mechanics, a group which is only represented projectively is still a quantum symmetry.

In fact, proceeding again formally, by iterating (16) infinitesimally using the commutation and association relations (13) and (14), and then integrating, we arrive at the corresponding global relations

$$
\begin{gathered}
\mathscr{P}_{w} \mathscr{P}_{v}=\mathrm{e}^{\mathrm{i} \Phi_{2}(x ; v, w)} \mathscr{P}_{v+w}, \\
\mathscr{P}_{w}\left(\mathscr{P}_{v} \mathscr{P}_{u}\right)=\mathrm{e}^{\mathrm{i} \Phi_{3}(x ; u, v, w)}\left(\mathscr{P}_{w} \mathscr{P}_{v}\right) \mathscr{P}_{u} .
\end{gathered}
$$

Here $\Phi_{2}(x ; v, w)$ is given by the integral of the two-form $\rho$ through the oriented two-simplex $\triangle^{2}(x ; v, w)$ based at $x$ and spanned by the translation vectors $v$ and $w$ in $M=\mathbb{R}^{d}$, while $\Phi_{3}(x ; u, v, w)$ is given by the integral of the three-form $H=\mathrm{d} \rho$ through the oriented three-simplex
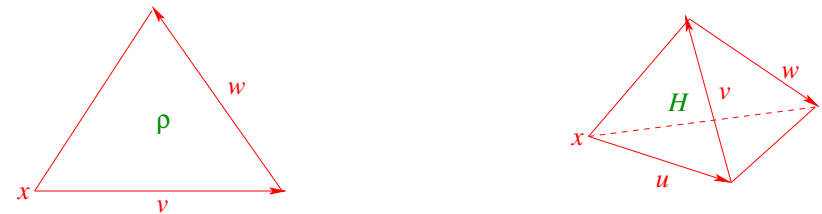

Figure 1 The two-simplex $\triangle^{2}(x ; v, w)$ on the left, over which the magnetic field $\rho$ is integrated, and the three-simplex $\triangle^{3}(x ; u, v, w)$ on the right, over which the magnetic charge $H$ is integrated.

$\triangle^{3}(x ; u, v, w)$ based at $x$ and spanned by $u, v$ and $w$ (see Figure 1). In the $d=3$ example from Section 2.2, the quantity $\Phi_{2}$ is interpreted as the 'magnetic flux' through the triangle $\triangle^{2}$ and $\Phi_{3}$ as the 'magnetic charge' enclosed by the tetrahedron $\triangle^{3}$. Since $H=\mathrm{d} \rho$, we expect that the phase factor $\Phi_{3}$ defines a (trivial) three-cocycle in a certain group cohomology of the translation group $T$. These naive considerations go back to [14] (see also [15]) in the context of nonassociativity of symmetry operations in quantum field theory, which lead to anomalies. Although this suggestion turns out to be heuristically correct in the field of a magnetic monopole, it has only been recently established in full generality at a rigorous level through considerations of higher quantization. In particular, in the following we shall address the following open questions which were not addressed in the original treatment of [14]:

i) How does one properly define the magnetic translation operators (15)?

ii) What is the precise definition of the representation of the translation group $T=\mathbb{R}^{d}$ given by (17)?

\subsection{Quantization I: $H=0$}

The questions posed above have well-known geometric answers in the case that $\mathrm{d} \rho=0$, which we shall now review. In this case the two-form $\rho=\mathrm{d} A$ can be written in terms of a global one-form $A \in \Omega^{1}(M)$ on configuration space, called a 'vector potential', and we may identify $\rho=F_{\nabla}$ as the curvature of a connection $\nabla^{L}$ on a (trivial) complex line bundle $L$ over $M=\mathbb{R}^{d}$. In this simple case the geometrical interpretation in terms of line bundles is somewhat superfluous, but it has the advantage that the treatment will generalize below to the more complicated situations we are ultimately interested in. In this case the magnetic Poisson brackets (4) generate an associative algebra and 
they can be represented by the (unbounded) operators

$$
\mathscr{O}_{x}=x,
$$$$
\mathscr{O}_{p}=-\mathrm{i} \hbar \nabla^{L}=-\mathrm{i} \hbar \mathrm{d}+A,
$$

acting on the quantum Hilbert space $\mathscr{H}=\mathrm{L}^{2}(M, L)$ of square-integrable sections of $L$ (equivalently squareintegrable functions on $M$ in this case). This is of course just the usual prescription in standard geometric quantization of the symplectic manifold $\left(\mathscr{M}, \sigma_{\rho}\right)$, with polarization given by the integrable distribution $T M$ which foliates the phase space $\mathscr{M}$ by leaves which are the Lagrangian submanifolds $M \hookrightarrow \mathscr{M}$, the image of the zero section of the cotangent bundle $\mathscr{M}=T^{*} M$.

Magnetic translations in this formulation have a natural geometric definition as parallel transport in the line bundle $L$ : For any section $\psi \in \mathscr{H}$, the magnetic translation operators (15) can be defined by

$\left(\mathscr{P}_{\nu} \psi\right)(x)=\exp \left(-\frac{\mathrm{i}}{\hbar} \int_{\triangle^{1}(x ; v)} A\right) \psi(x-v)$,

where $x \in M, v \in T$, and $\Delta^{1}(x ; v)$ is the oriented onesimplex based at $x$ along the vector $v$, i.e. the straight line from $x-v$ to $x$ (see Figure 2). Explicit computation using $\mathrm{d} A=\rho$ and Stokes' Theorem shows that they define a (trivial) weak projective representation of the translation group $T=\mathbb{R}^{d}$ on the Hilbert space $\mathscr{H}$ with

$\left(\mathscr{P}_{w} \mathscr{P}_{\nu} \psi\right)(x)=\omega_{v, w}(x)\left(\mathscr{P}_{\nu+w} \psi\right)(x)$,

where

$\omega_{\nu, w}(x)=\exp \left(-\frac{\mathrm{i}}{\hbar} \int_{\triangle^{2}(x ; w, v)} \rho\right)$.

The adjective 'weak' refers to the fact that (21) satisfies a twisted form of the usual cocycle condition given by

$\omega_{\nu, w}(x-u) \omega_{u+v, w}^{-1}(x) \omega_{u, v+w}(x) \omega_{v, w}^{-1}(x)=1$,

and so defines a two-cocycle on the translation group $T$ with values in $C^{\infty}(M, \mathrm{U}(1))$, the $\mathrm{U}(1)$-valued functions on $M$. In the special case where the magnetic field $\rho$ is constant, i.e. the component functions $\rho_{i j}$ are constant, the two-cocycle (21) simplifies to the constant phase

$\omega_{\nu, w}=\mathrm{e}^{-\frac{\mathrm{i}}{2 \hbar} \rho(\nu, w)}$,

and so defines a two-cocycle in the group cohomology $H^{2}(T, \mathrm{U}(1))$; in this instance, the magnetic translation operators generate an ordinary projective representation of $T$ on $\mathscr{H}$.

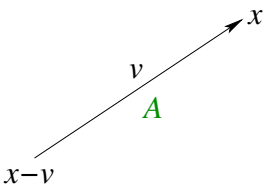

Figure 2 The one-simplex $\Delta^{1}(x ; v)$ along which the vector potential $A$ is integrated.

In this class of magnetic Poisson structures it is also possible to give the quantization map $f \longmapsto \mathscr{O}_{f}$ explicitly in terms of the magnetic Weyl transform which sends a phase space function $f \in C^{\infty}(\mathscr{M})$ to an operator $\mathscr{O}_{f} \in$ $\operatorname{End}(\mathscr{H})$. For this, one introduces the magnetic Weyl system which is the family of bounded operators ${ }^{1}$

$W(x, p): \mathscr{H} \longrightarrow \mathscr{H}$

parameterized by points $X=(x, p) \in \mathscr{M}$ and defined using the magnetic translation operators by

$(W(x, p) \psi)(y)=\mathrm{e}^{\frac{\mathrm{i} \hbar}{2} p \cdot x} \mathrm{e}^{-\mathrm{i} p \cdot y}\left(\mathscr{P}_{x} \psi\right)(y)$.

The quantization map is then defined by the bounded operator

$\mathscr{O}_{f}=\int_{\mathscr{M}}\left(\int_{\mathscr{M}} \mathrm{e}^{\mathrm{i} \sigma_{0}(X, Y)} f(Y) \frac{\mathrm{d} Y}{(2 \pi)^{d}}\right) W(X) \frac{\mathrm{d} X}{(2 \pi)^{d}}$,

where $\mathrm{d} X$ is the Lebesgue measure on $\mathscr{M}$. This defines an invertible linear transformation and the pre-image of the operator product $\mathscr{O}_{f} \mathscr{O}_{g}$ defines the magnetic Moyal-Weyl star product $f \star_{\rho} g$ on $C^{\infty}(\mathscr{M})$ through $\mathscr{O}_{f \star_{\rho} g}:=\mathscr{O}_{f} \mathscr{O}_{g}$; explicitly, it can be written in terms of the two-cocycle (21) as an oscillatory integral

$$
\begin{aligned}
& \left(f \star_{\rho} g\right)(X)= \\
& =\frac{1}{(\pi \hbar)^{d}} \int_{\mathscr{M}} \int_{\mathscr{M}} \mathrm{e}^{-\frac{2 \mathrm{i}}{\hbar} \sigma_{0}(Y, Z)} \omega_{x+y-z, x-y+z}(x-y-z) \\
& \times f(X-Y) g(X-Z) \mathrm{d} Y \mathrm{~d} Z .
\end{aligned}
$$

\footnotetext{
1 Here we are glossing over many technical functional analytic details: The domain of the magnetic Weyl transform is smaller than the smooth functions $C^{\infty}(\mathscr{M})$ and the Weyl operators $W(x, p)$ only act on a Schwartz subspace of $\mathscr{H}$ in a suitable way; see e.g. [16] for the precise treatment. These details are not important for the discussion that follows.
} 
This is a convergent star product (on a suitable algebra of Schwartz functions) and so defines a strict deformation quantization of the magnetic Poisson structure on $\mathscr{M}$. In the case that $\rho$ is constant, it can be simplified to the usual Moyal-Weyl form of a twisted convolution product

$$
\begin{aligned}
& (f \star \rho g)(X)= \\
& =\frac{1}{(\pi \hbar)^{d}} \int_{\mathscr{M}} \int_{\mathscr{M}} \mathrm{e}^{-\frac{2 \mathrm{i}}{\hbar} \sigma_{\rho}(Y, Z)} f(X-Y) g(X-Z) \mathrm{d} Y \mathrm{~d} Z .
\end{aligned}
$$

This demonstrates another importance of the magnetic translation operators: They provide a bridge between geometric quantization and deformation quantization. This is the starting point for a reformulation of canonical quantum mechanics as phase space quantum mechanics, see e.g. [8] and [17] for reviews in the contexts of magnetic monopole physics and non-geometric string theory, respectively.

\subsection{Quantization II: $H \neq 0$}

Let us now turn to the quantization problem in our main case of interest, when $H=\mathrm{d} \rho \neq 0$. In this case, the technical and conceptual problem is that the operator/state formulation of geometric quantization discussed above cannot handle nonassociative magnetic Poisson algebras: Operators which act on a separable Hilbert space always associate.

The exception is the case of a singular point distribution of magnetic charge. Recalling that the magnetic field (8) of a Dirac monopole is defined on $M^{\circ}:=\mathbb{R}^{3} \backslash\{\mathbf{0}\}$, we can consider the quantization problem on the topologically non-trivial domain $M^{\circ}$ outside the support of the magnetic charge distribution; physically, this is tantamount to saying that the electric charge never reaches, and the wavefunctions vanish at, the location of the monopole. In this case the magnetic Poisson algebra is associative on $M^{\circ}$, and the magnetic field $\rho=\frac{e}{\hbar} \mathrm{d} A_{\mathrm{D}}$ admits locally defined vector potentials (9) which can be glued together by a gauge transformation between the two Dirac string singularities along the unit vectors $\mathbf{n}$ and $-\mathbf{n}$, corresponding to the northern and southern hemispheres of the homotopy equivalent $S^{2} \simeq M^{\circ}$. The two-form $\rho$ can then be identified as the curvature of a connection $\nabla^{L}$ on a non-trivial line bundle $L \longrightarrow M^{\circ}$ of first Chern class

$n=\frac{2 e g}{\hbar}$

if and only if $n \in \mathbb{Z}$. This is the famous Dirac charge quantization condition, and its present geometric derivation goes back to [18]. Then the quantum Hilbert space of geometric quantization is $\mathscr{H}=\mathrm{L}^{2}\left(M^{\circ}, L\right)$. The magnetic Weyl transform in this instance on $\mathscr{M}^{\circ}=T^{*} M^{\circ}$ can be constructed as before using magnetic translation operators defined through parallel transport in the line bundle $L$, which associate due to the quantization condition (29), and as previously it induces an associative phase space star product [19]. Hence in this case too we find an explicit form for the quantization map $f \longmapsto \mathscr{O}_{f}$, and hence a natural correspondence between geometric quantization and deformation quantization approaches.

On the other hand, for generic smooth distributions $H \in \Omega^{3}(M)$, standard geometric quantization breaks down. Even if the support of $H$ is a compact submanifold of $M$, one cannot consider the quantization problem on the complement $M^{\circ}$ in the way we did above: The quantization condition on the first Chern class (29) would be violated by continuous deformations of $H$ in its support, and hence no quantum line bundle for geometric quantization exists in this case. In particular, for a uniform distribution of magnetic charge throughout $M$, removing the support would leave $M^{\circ}=\varnothing$. Hence we must resort to alternative approaches to the quantization of generic magnetic Poisson structures. In the remainder of this contribution we discuss three such perspectives on this quantization problem, and how they address the questions raised in Sections 2.2-2.4 above.

\section{Perspective l: deformation quantization}

The most straightforward way to quantize a general magnetic Poisson structure is via Kontsevich's formalism for deformation quantization of Poisson manifolds [20], which can be carried out without any reference to a Hilbert space formulation. At the heart of Kontsevich's formalism is the Formality Theorem, which provides an $L_{\infty}$-quasi-isomorphism from the $L_{\infty}$-algebra of multivector fields, equipped with the Schouten bracket, to the $L_{\infty}$-algebra of multidifferential operators, equipped with the Gerstenhaber bracket. This formalism can also be extended to quantize twisted Poisson structures using algebroid stacks [21-23].

In the setting of magnetic Poisson structures, which was first worked out explicitly in [12], Kontsevich's formality maps provide a noncommutative and nonassociative star product on the algebra of formal power series $C^{\infty}(\mathscr{M})[[\hbar]]$ for any twisting three-form $H=\mathrm{d} \rho \in \Omega^{3}(M)$. It is given by

$f \star_{H} g=f g+\frac{\mathrm{i} \hbar}{2}\{f, g\}_{\rho}+\sum_{n \geqslant 2} \frac{(\mathrm{i} \hbar)^{n}}{n !} \mathrm{b}_{n}(f, g)$, 


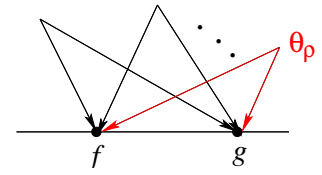

Figure 3 Graphical representation of the contributions to $\mathrm{b}_{n}(f, g)$. There are $n$ insertions of the bivector $\theta_{\rho}$ in each diagram.

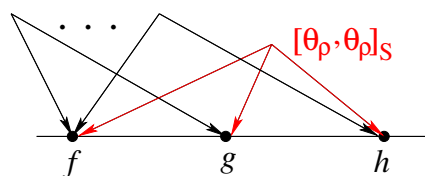

Figure 4 Graphical representation of the contributions to $\mathrm{t}_{n}(f, g, h)$. There is a single insertion of the trivector $\left[\theta_{\rho}, \theta_{\rho}\right]_{S}$ and $n-1$ insertions of the bivector $\theta_{\rho}$ in each diagram.

where $\mathbf{b}_{n}=U_{n}\left(\theta_{\rho}, \ldots, \theta_{\rho}\right)$ are bidifferential operators determined by the bivector $\theta_{\rho}$. Here $U_{n}$ denote the formality maps which generally send a collection of $n$ multivector fields to multidifferential operators, and the functions $\mathrm{b}_{n}(f, g)$ can be computed by a combinatorial algorithm which sums over graphs with prescribed weight given by suitable integrals over the upper hyperbolic half-plane $\mathbb{U}$, that are represented diagrammatically by applying the legs of the bivector insertions $\theta_{\rho}$ to the functions $f$ and $g$ sitting on the boundary $\mathbb{R}$ of $\mathbb{U}$ (see Figure 3 ).

In addition to providing a deformation quantization of the classical magnetic Poisson brackets (3) in the sense of (12), the Kontsevich formality maps further give a deformation quantization of the three-bracket defined by the classical Jacobiator (6). This quantum three-bracket is given by

$[f, g, h]_{\star_{H}}=-\hbar^{2}\{f, g, h\}_{\rho}+\sum_{n \geqslant 3} \frac{(\mathrm{i} \hbar)^{n}}{n !} \mathrm{t}_{n}(f, g, h)$,

where $\mathrm{t}_{n}=U_{n+1}\left(\left[\theta_{\rho}, \theta_{\rho}\right]_{S}, \theta_{\rho}, \ldots, \theta_{\rho}\right)$ are tridifferential operators, and the contributions $\mathrm{t}_{n}(f, g, h)$ can be computed by an analogous combinatorial algorithm in terms of the trivector $\left[\theta_{\rho}, \theta_{\rho}\right]_{S}$ which is depicted in Figure 4.

In the general case, the star product (30) is an asymptotic series in $\hbar$ and defines a formal deformation quantization of the magnetic Poisson structure. It is difficult to compute in generality beyond the first few non-trivial orders in $\hbar$. However, for $H$ constant, and with the choice of magnetic field $\rho_{i j}(x)=\frac{1}{3} H_{i j k} x^{k}$, the expansion simplifies enormously: Most diagrams vanish and all nonvanishing contributions factorize as powers of a single graph [12]. The resulting series can be formally summed to all orders in $\hbar$ and rewritten using Fourier transformations as a twisted convolution product [24]

$$
\begin{aligned}
& \left(f \star_{H} g\right)(X)= \\
& =\frac{1}{(\pi \hbar)^{d}} \int_{\mathscr{M}} \int_{\mathscr{M}} \mathrm{e}^{-\frac{2 \mathrm{i}}{\hbar} \sigma_{\rho}(Y, Z)} f(X-Y) g(X-Z) \mathrm{d} Y \mathrm{~d} Z .
\end{aligned}
$$

Note that this formula is formally identical to the associative Moyal-Weyl type star product (28) which was written in the case of constant $\rho$. Here $\rho$ is not constant and the formula defines a convergent nonassociative star product (on a suitable algebra of Schwartz functions), giving a strict deformation quantization of the magnetic Poisson structure in this instance.

In this framework, the quantization map is simply $\mathscr{O}_{f}=f$ with the multiplication on $C^{\infty}(\mathscr{M})[[\hbar]]$ given by the star product (30). The nonassociative magnetic translation operators (15) are thus given by the functions

$\mathscr{P}_{v}:=\mathrm{e}^{\frac{\mathrm{i}}{\hbar} p \cdot v}$.

For $H$ constant, an explicit computation using the exact formula (32) shows that the higher projective representation (17) of the translation group $T=\mathbb{R}^{d}$ is determined by a three-cocycle $\omega_{u, v, w}$ in the group cohomology $H^{3}(T, \mathrm{U}(1))$ with

$$
\begin{aligned}
\mathscr{P}_{v} \star_{H} \mathscr{P}_{w} & =\Pi_{v, w}(x) \mathscr{P}_{v+w}, \\
\left(\mathscr{P}_{u} \star_{H} \mathscr{P}_{v}\right) \star_{H} \mathscr{P}_{w} & =\omega_{u, v, w} \mathscr{P}_{u} \star_{H}\left(\mathscr{P}_{v} \star_{H} \mathscr{P}_{w}\right),
\end{aligned}
$$

where

$\Pi_{v, w}(x)=\mathrm{e}^{-\frac{\mathrm{i}}{6 \hbar} H(x, v, w)}$

is a two-cochain with constant coboundary

$\omega_{u, v, w}=\mathrm{e}^{\frac{\mathrm{i}}{6 \hbar} H(u, v, w)}$.

This approach thus answers the questions raised at the end of Section 2.4. The perspective on nonassociativity in terms of three-cocycles in the group cohomology $H^{3}(T, \mathrm{U}(1))$ was emphasised by [14, 12,25]. They can also be interpreted as three-cocycles of a suitable quasi-Hopf algebra in a cochain twist approach to deformation quantization [24].

It can be shown that the phase space formulation of nonassociative quantum mechanics in this setting, for 
constant magnetic charge $H$ or constant $R$-flux, is physically sensible and gives novel testable quantitative predictions [24] (see e.g. [8, 17] for reviews). This answers the questions raised at the end of Sections 2.2 and 2.3 in this particular instance. On the other hand, the approach suffers from many problems. For example, the quantization is formal in $\hbar$ for non-constant $H$, so that for generic distributions of magnetic charge the deformation parameter cannot be identified with the physical Planck constant; as usual, deformation quantization is not a genuine quantization from this perspective. Moreover, there are the usual technical and conceptual issues associated with phase space quantum mechanics (see e.g. [8, 17]). Finally, the loss of the Leibniz rule for the commutator constructed using the nonassociative star product casts doubt on the existence of suitable integrals of motion which enable at least partial integrability of the dynamical system. In light of these drawbacks, we would like to seek a framework which avoids the deficiencies of deformation quantization and takes us closer to a framework akin to an operator/state correspondence in geometric quantization. This is the goal of our next two perspectives.

\section{Perspective II: symplectic realization}

One way to approach the problem of a Hilbert space formulation for the quantization of magnetic Poisson structures is to generalize the well-known technique of symplectic realization in Poisson geometry, which allows one to cast the quantization of generic Poisson manifolds into the framework of standard geometric quantization of symplectic manifolds. A symplectic realization of a Poisson structure $\theta$ on a manifold $M$ is a symplectic manifold $(S, \Omega)$ together with a surjective submersion $S \longrightarrow M$ which is a Poisson map. The original local construction goes back to [26], while a global formulation is given in $[27,28]$ using the corresponding symplectic groupoid which integrates the Poisson manifold $(M, \theta)$. A global generalization integrating twisted Poisson structures in terms of almost symplectic manifolds is given in [29].

A local symplectic realization of the magnetic Poisson algebra is constructed in [30]. Concretely, it "doubles" $\mathscr{M}$ to an extended phase space $\mathscr{S}$ with local coordinates $\left(x^{i}, \tilde{x}^{i}, p_{i}, \tilde{p}_{i}\right)$ using local Darboux coordinates $\left(x^{i}, \pi_{i}\right)$ and $\left(\tilde{x}^{i}, \tilde{\pi}_{i}\right)$ with the generalized Bopp shifts $p_{i}=$ $\pi_{i}-\frac{1}{2} \rho_{i j}(x) \tilde{x}^{j}$ and $\tilde{p}_{i}=\tilde{\pi}_{i}$. Then the symplectic algebra of coordinate functions

$\left\{x^{i}, p_{j}\right\}=\left\{\tilde{x}^{i}, p_{j}\right\}=\left\{x^{i}, \tilde{p}_{j}\right\}=\delta^{i}{ }_{j}$,

$\left\{p_{i}, p_{j}\right\}=\rho_{i j}(x)+\frac{1}{2} \tilde{x}^{k}\left(\frac{\partial \rho_{i j}(x)}{\partial x^{k}}-H_{i j k}(x)\right)$,

$\left\{p_{i}, \tilde{p}_{j}\right\}=\left\{\tilde{p}_{i}, p_{j}\right\}=\frac{1}{2} \rho_{i j}(x)$

is by construction an associative algebra. The pullback of the corresponding symplectic form $\Omega$ on $\mathscr{S}$ by the zero section of the projection $\mathscr{S} \longrightarrow \mathscr{M}$ coincides with the almost symplectic form $\sigma_{\rho}$. The integrability of this realization is not yet understood, though it is natural to speculate that it may be related to the integration of Lie bialgebroids to Poisson-Lie groupoids discussed in [31]. A local generalization to arbitrary quasi-Poisson structures is discussed in [32].

This local symplectic realization is intimately related to the approach via deformation quantization discussed in Section 3. The quantization of the algebra (38a) on $C^{\infty}(\mathscr{M})$ via the Schrödinger polarization

$\widehat{\tilde{p}}_{i}=\mathrm{i} \hbar \frac{\partial}{\partial x^{i}}$,
$\widehat{\widetilde{x}}^{i}=-\mathrm{i} \hbar \frac{\partial}{\partial p_{i}}$

coincides with the associative composition algebra of differential operators $\left(\operatorname{Diff}(\mathscr{M})[[\hbar]], \circ_{H}\right)$ on the original phase space $\mathscr{M}$ which governs observables in nonassociative quantum mechanics [24]. Here for phase space functions $f, g \in C^{\infty}(\mathscr{M})$, the composition product ${ }^{\circ}{ }_{H}$ is defined through

$\left(f \circ{ }_{H} g\right) \star_{H} \varphi:=f \star_{H}\left(g \star_{H} \varphi\right)$

for arbitrary test functions $\varphi \in C^{\infty}(\mathscr{M})$, and in general $f \circ_{H} g$ is not a function but a formal power series in $\hbar$ of differential operators [30].

One can now ask how to reduce the extended dynamical system (at both classical and quantum levels) in a consistent way so as to recover the original magnetic Poisson manifold. This can be analysed by introducing the $\mathrm{O}(d, d) \times \mathrm{O}(d, d)$-invariant Hamiltonian given by

$\mathrm{Ham}=p_{I} \eta^{I J} p_{J}$,

where $\left(p_{I}\right)=\left(p_{i}, \tilde{p}_{i}\right)$ and

$\eta=\left(\begin{array}{cc}0 & 1_{d} \\ 1_{d} & 0\end{array}\right)$

is the constant $\mathrm{O}(d, d)$-invariant metric. With this choice, Hamilton's equations of motion with the non-degenerate 
Poisson brackets (38a) reproduces, in the $d=3$ example of Section 2.2, the correct Lorentz force law for the physical coordinates $(x, p)$. The formalism is thus reminiscent of an approach based on double field theory. In contrast, however, it is not possible to impose here a section constraint that eliminates the unwanted degrees of freedom resulting from the doubling. In [30] it is shown that a consistent Hamiltonian reduction of the symplectic realization can eliminate the auxiliary coordinates $(\tilde{x}, \tilde{p})$ if and only if $H=0$ : There is no polarization of the extended symplectic algebra which is consistent with both the Lorentz force and the original nonassociative magnetic Poisson algebra.

The problem with the approach based on symplectic realization thus remains in the physical meaning of the spurious degrees of freedom. At first glance an interpretation may be given by looking at the dynamics in $d=3$ dimensions: For constant magnetic charge $H$, the Lorentz force is equivalent to the equations of motion of an electric charge in the background of a Dirac monopole field (8) with additional frictional forces [25]. A dissipative dynamical system requires the introduction of extra degrees of freedom representing a reservoir if one wishes to conserve the total energy. However, in the present case the Hamiltonian (40) is automatically an integral of motion and so there is no need to introduce new variables in order to conserve the energy. It is therefore not clear what the extra coordinates $(\tilde{x}, \tilde{p})$ mean in the symplectic realization of the magnetic Poisson structure. Moreover, the extra variables hide the interesting consequences of nonassociativity (by construction), such as the three-cocycles characterizing the higher projective representation defined by magnetic translation operators; see [30] for a discussion of this point. We therefore need to appeal to some sort of Hilbert space formalism which can tackle the nonassociativity of magnetic Poisson brackets head on. This is the topic of our third and final perspective.

\section{Perspective III: higher geometric quantization}

Our considerations above motivate the desire to deal with nonassociative algebras directly, which can be achieved through higher structures: Whereas nonassociativity forbids the definition of magnetic translations as operators on a separable Hilbert space, as linear representations of groups are always associative, nonassociativity can occur when representing elements of a group by endofunctors of a symmetric monoidal category; the multiplication law may then close only up to a natural isomorphism and the natural isomorphisms in turn may only be represented up to higher projective phases. In the following we shall refer to these "representations" as "weak projective 2-representations'. In other words, we capture nonassociativity by working in a more general category than the category of vector spaces normally used in physics. We pursue this line of approach by categorifying the framework for geometric quantization discussed in Section 2.5: We replace Hilbert spaces of sections of line bundles for $\mathrm{d} \rho=0$ with 2-Hilbert spaces of sections of a suitable geometric object which encodes non-trivial magnetic charge $H=\mathrm{d} \rho \neq 0$. Specifically, we provide a natural geometric definition of nonassociative magnetic translations by realizing them as parallel transport functors on a bundle gerbe $\mathscr{I}_{\rho}$ canonically associated with the magnetic field two-form $\rho$ on $\mathbb{R}^{d}$.

\subsection{Bundle gerbes}

We begin by discussing what the suitable higher version of a line bundle should be in order to pursue our quantization scheme. Let $\pi: Y \longrightarrow M$ be a surjective submersion over a manifold $M$; for example, we can take $Y$ to be the total space of an open cover of $M$. Then the $p$-fold fibre products $Y^{[p]}:=Y \times_{M} \cdots \times_{M} Y$ form a simplicial space with face maps $\pi_{i}: Y^{[p]} \longrightarrow Y^{[p-1]}$ defined by omitting the $i$-th entry of a $p$-tuple for $i=1, \ldots, p$; for example, $Y^{[p]}$ can be the space of $p$-fold intersections of open sets of a cover of $M$. For $p=2$ this defines the pair groupoid $Y^{[2]} \rightrightarrows Y$ whose source and target maps are $\pi_{2}$ and $\pi_{1}$, respectively, and whose orbit space is the base manifold $M$ itself. Then a bundle gerbe $(L, Y)$ on $M$ is a groupoid central extension of $Y^{[2]} \rightrightarrows Y$ [33]; it can be depicted by

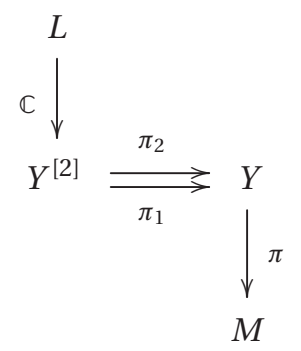

Here we assume that $L$ is a complex line bundle over $Y^{[2]}$ in order to extend our previous considerations, but the definition also holds at the level of principal U(1)-bundles. The groupoid multiplication $\left(y_{1}, y_{2}\right) \circ\left(y_{2}, y_{3}\right)=\left(y_{1}, y_{3}\right)$ on $Y^{[2]} \rightrightarrows Y$ additionally gives a bundle gerbe multiplication which is a bundle isomorphism $\mu: \pi_{3}^{*}(L) \otimes \pi_{1}^{*}(L) \stackrel{\simeq}{\longrightarrow} \pi_{2}^{*}(L)$ 
over $Y^{[3]}$, and can be depicted by

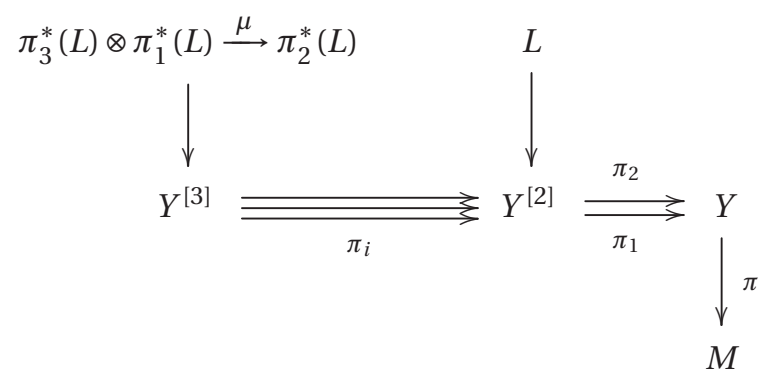

The bundle gerbe multiplication is associative over $Y^{[4]}$.

A connection on a bundle gerbe $(L, Y)$ is a connection $\nabla^{L}$ on the line bundle $L \longrightarrow Y^{[2]}$ together with a two-form $\rho \in \Omega^{2}(Y)$ satisfying $\pi_{2}^{*}(\rho)-\pi_{1}^{*}(\rho)=F_{\nabla^{L}}$. By the Bianchi identity $\mathrm{d} F_{\nabla L}=0$, the three-form $\mathrm{d} \rho$ on $Y$ descends to a closed three-form $H \in \Omega^{3}(M)$, with $\pi^{*} H=\mathrm{d} \rho$, which is called the curvature of the bundle gerbe. Analogously to the first Chern class for line bundles, the curvature $H$ is a de Rham representative of a higher characteristic class in $H^{3}(M, \mathbb{Z})$ measuring obstructions to topological triviality of the bundle gerbe (in a suitable sense that we do not spell out here), called the Dixmier-Douady class. It is clear then that bundle gerbes with connection are the appropriate receptacle to describe a geometric approach to the quantization of generic magnetic Poisson structures.

\subsection{Sections of bundles gerbes}

To define sections of bundle gerbes, we recall that Hermitian vector bundles (with connection) on $M$ are objects in a symmetric monoidal category $\operatorname{HVbdl}(M)$ under tensor product of vector bundles; under the direct sum of vector bundles, it moreover has the structure of a rig category, the categorification of a ring without additive inverses. A section of a vector bundle $V \longrightarrow M$ can be equivalently regarded as a morphism in this category from the trivial vector bundle $I_{0}$ with connection to $V$, and in particular a morphism from the trivial bundle $I_{0}$ to itself is the same thing as a function on $M$; the $C^{\infty}(M)$-module structure of $\Gamma(M, V)$ translates into an action of the latter space of morphisms on the former via composition of morphisms. Under the embedding of $\mathbb{C}$ into the morphisms as the constant functions on $M$, this makes $\operatorname{HVbdl}(M)$ into a $\mathbb{C}$-linear category.

Analogously, bundle gerbes $\mathscr{G}=(L, Y)$ (with connection) on $M$ are objects in a symmetric monoidal 2category. The original construction is due to [34], and was subsequently extended by [2] to show that the 2- category in question has the further structure of a closed abelian symmetric monoidal category enriched in symmetric monoidal categories. A section of a bundle gerbe $\mathscr{G}=(L, Y)$ is is a (left) module over $\mathscr{G}$, defined by a vector bundle $E$ over $Y$ and an action $L \otimes \pi_{1}^{*} E \longrightarrow \pi_{2}^{*} E$ which is an isomorphism of bundles over $Y^{[2]}$ satisfying the obvious associativity constraint on $Y^{[3]}$; this is the same thing as a 1-morphism from the trivial bundle gerbe $\mathscr{I}_{0}$ with connection to $\mathscr{G}$. Then the 2-Hilbert space of sections $\Gamma(M, \mathscr{G})$ is defined to be the Hilb-module category of morphisms $\mathscr{I}_{0} \longrightarrow \mathscr{G}$, where Hilb is the symmetric monoidal category of finite-dimensional complex Hilbert spaces under tensor product. ${ }^{2}$ The category $\Gamma(M, \mathscr{G})$ enjoys the following properties:

i) It carries the structure of a rig module category over the rig category $\operatorname{HVbdl}(M)$.

ii) There is an inner product bifunctor $\langle\rangle:, \Gamma(M, \mathscr{G})^{\mathrm{op}} \times$ $\Gamma(M, \mathscr{G}) \longrightarrow$ Hilb.

See $[2,3]$ for precise definitions and further details. In this categorification the ground field $\mathbb{C}$ is replace with the rig category Hilb and the ring of functions $C^{\infty}(M)$ is replaced with the rig category $\operatorname{HVbdl}(M)$.

The 2-Hilbert space $\Gamma(M, \mathscr{G})$ admits a particularly simple description on $M=\mathbb{R}^{d}$, in which case we can restrict attention to topologically trivial bundle gerbes $\mathscr{G}=\mathscr{I}_{\rho}$ with connection specified entirely by a globally defined two-form $\rho \in \Omega^{2}(M)$. The groupoid central extension (42) can then be replaced with the simpler extension

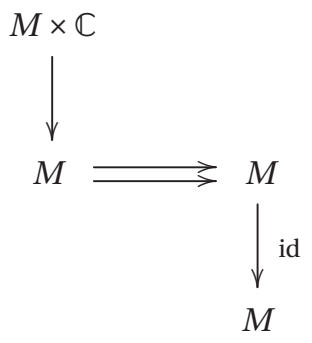

and up to equivalence the category $\Gamma(M, \mathscr{G})$ admits the following concrete description: ${ }^{3}$

2 The restriction to finite-dimensional Hilbert spaces and vector bundles of finite rank restricts to bundle gerbes $\mathscr{G}$ which have torsion Dixmier-Douady class. This restriction will be irrelevant for our considerations below, as we shall always work with trivial bundle gerbes over $M=\mathbb{R}^{d}$. See [2] for a more indepth discussion of this issue.

3 This is not a generic description of the 2-Hilbert space in the topologically trivial case $[H]=0$, but rather of a full subcate- 
i) Its objects are topologically trivial Hermitian vector bundles with connection on $M$, i.e. globally defined one-forms $\eta \in \Omega^{1}(M, \mathrm{u}(n))$ valued in Hermitian $n \times n$ matrices for any $n \in \mathbb{N}_{0}$.

ii) Its morphisms are parallel morphisms of vector bundles with connection on $M$, i.e. a morphism $f: \eta \longrightarrow$ $\eta^{\prime}$ is an $n \times n^{\prime}$ matrix-valued function $f: M \longrightarrow$ $\operatorname{Mat}\left(n \times n^{\prime}\right)$ satisfying i $\eta^{\prime} f=\mathrm{i} f \eta-\mathrm{d} f$. This resembles a gauge transformation except that $f$ need not be invertible: In the construction of the 2-Hilbert space the non-invertible 1-morphisms are essential, and these correspond to the matrix-valued functions of size $n \times n^{\prime}$ with $n \neq n^{\prime}$.

\subsection{Magnetic translation functors}

A precise definition of nonassociative magnetic translations was constructed in [16] on the 2-Hilbert space $\Gamma\left(M, \mathscr{I}_{\rho}\right)$ in terms of the parallel transport functor $\mathscr{P}_{v}$ : $\Gamma\left(M, \mathscr{I}_{\rho}\right) \longrightarrow \Gamma\left(M, \mathscr{I}_{\rho}\right)$ which is defined on objects $\eta$ as an infinitesimal version of the parallel transport operators (19) and by the usual action of the translation group $T=\mathbb{R}^{d}$ on morphisms $f$ via pullback $v^{*}(f)$ :

$$
\begin{aligned}
\left.\mathscr{P}_{v}(\eta)\right|_{x}(a) & =\left.\eta\right|_{x-v}(a)+\frac{1}{\hbar} \int_{\triangle^{1}(x ; v)} \iota_{a} \rho, \\
\mathscr{P}_{v}(f)(x) & =f(x-v),
\end{aligned}
$$

where $v \in T, x \in M$ and $\iota_{a}$ denotes contraction with the vector $a \in \mathbb{R}^{d}$. This definition can be understood by transgressing the gerbe $\mathscr{I}_{\rho}$ to a line bundle with connection over the loop space $L M$ of $M$ [2], and defining parallel transport over $L M$ in the usual way. That is, $\mathscr{P}_{v}$ translates a one-form $\eta$ by $v$ and adds the one-form obtained by integrating $\rho$ along the one-simplex $\Delta^{1}(x ; v)$; the extra term is necessary in the parallel condition in order to induce an additional one-form coming from the integration of $H=\mathrm{d} \rho$ over a two-simplex, which is the incarnation of the transgression line bundle over the boundary of a twosimplex relating parallel transport along two paths with the same endpoints. This defines a weak module functor

gory defined by the trivializations of the line bundle $L \longrightarrow Y^{[2]}$ and surjective submersion $\pi: Y \longrightarrow M$ indicated in (44), which is sufficient for the present purposes; see [16] for a more detailed discussion of this point. For notational ease, we continue to use the same symbol $\Gamma\left(M, \mathscr{I}_{\rho}\right)$ for this subcategory. in the sense that

$\mathscr{P}_{\nu}(\xi \otimes \eta)=v^{*}(\xi) \otimes \mathscr{P}_{\nu}(\eta)$

for $\xi \in \Omega^{1}(M, \mathrm{u}(k))$.

The magnetic translation functors are subjected to natural coherence isomorphisms

$\Pi_{v, w}: \mathscr{P}_{v} \circ \mathscr{P}_{w}=\chi_{v, w} \otimes \mathscr{P}_{v+w}$

where

$\left.\chi_{v, w}\right|_{x}(a)=\frac{1}{\hbar} \int_{\triangle^{2}(x ; w, v)} \iota_{a} H$

is a connection one-form of the trivial line bundle on $M$, which corresponds to the transgression line bundle over the two-simplex $\triangle^{2}(x ; w, v)$. The components of $\Pi_{v, w}$ are defined by

$\Pi_{v, w \mid \eta}(x):=\exp \left(-\frac{\mathrm{i}}{\hbar} \int_{\triangle^{2}(x ; w, v)} \rho\right)$.

'Nonassociativity' is a natural property of the coherence isomorphisms when applied to the two possible bracketings of a triple composition of parallel transport functors $\mathscr{P}_{u} \circ \mathscr{P}_{v} \circ \mathscr{P}_{w}$. By iterating (47a), this identifies natural transformations through

$\Pi_{u+v, w} \circ \Pi_{u, v}(x)=\omega_{u, v, w}(x) \Pi_{u, v+w} \circ \mathscr{P}_{u}\left(\Pi_{v, w}\right)(x)$

where

$\omega_{u, v, w}: \chi_{u+v, w} \otimes \chi_{u, v} \longrightarrow \chi_{u, v+w} \otimes u^{*}\left(\chi_{v, w}\right)$

is the morphism in $\Gamma\left(M, \mathscr{I}_{\rho}\right)$ defined by

$\omega_{u, v, w}(x):=\exp \left(\frac{\mathrm{i}}{\hbar} \int_{\triangle^{3}(x ; w, v, u)} H\right)$

It is these coherence identities which make precise the relations (17) among magnetic translations, and we shall discuss their precise representation theoretic meaning below. In the case that the magnetic charge $H$ is constant, the pertinent $\mathrm{U}(1)$-valued functions on $M$ simplify to

$$
\begin{aligned}
\Pi_{v, w \mid \eta}(x) & =\mathrm{e}^{-\frac{\mathrm{i}}{6 \hbar} H(x, v, w)}, \\
\omega_{u, v, w} & =\mathrm{e}^{\frac{\mathrm{i}}{6 \hbar} H(u, v, w)},
\end{aligned}
$$

which agrees with what we found in Section 3 in the approach based on deformation quantization.

In Section 3 the relations (34) showed that $\Pi_{v, w}$ and $\omega_{u, v, w}$ (for $H$ constant) have natural interpretations in the group cohomology $H^{3}(T, \mathrm{U}(1))$, and define a "higher" projective representation of the translation group $T$ in this 
sense. Here we can make this notion of higher projective representation more precise in the language of category theory. We do not spell out the general definitions, which can be found in [16], but simply use the properties discussed above to characterize these notions. Analogously to the $H=0$ case, $\omega_{u, v, w}$ define a three-cocycle on $\mathbb{R}^{d}$ with values in $C^{\infty}(M, \mathrm{U}(1))$. On the other hand, the pairs $\left(\chi_{v, w}, \omega_{u, v, w}\right)$ define a higher weak two-cocycle on $\mathbb{R}^{d}$ with values in the Hilb-algebra category $\operatorname{HVbdl}(M)$. These constructions were collected together in [16] to give the following central result.

Theorem 5.1. The pairs $\left(\mathscr{P}_{v}, \Pi_{v, w}\right)$ define a weak projective 2-representation of the translation group $\mathbb{R}^{d}$ on the $H \operatorname{Vbdl}(M)$-module category $\Gamma\left(M, \mathscr{I}_{\rho}\right)$, the 2-Hilbert space of sections of the bundle gerbe $\mathscr{I}_{\rho}$ on $M$.

This approach thus completely answers the questions which arose at the end of Section 2.4 in full generality. However, at this stage it is not clear how it addresses the issues raised in Sections 2.2 and 2.3, and there are many open issues which remain. In the framework of nonassociative quantum mechanics, it is not clear what is the physical significance of the 2 -Hilbert space $\Gamma\left(M, \mathscr{I}_{\rho}\right)$ in terms of states, observables, and so on; see [16] for some preliminary analysis along these lines, where it is also shown how the fake curvature condition of higher gauge theory naturally emerges in terms of this interpretation when considering covariant derivatives on these bundle gerbes. In this sense it would be interesting to develop a "higher magnetic Weyl transform" to determine the quantization map as a natural transformation from functions $f \in C^{\infty}(\mathscr{M})$, regarded as objects in the functor category $[\mathscr{M} \rightrightarrows \mathscr{M}, \mathbb{C} \rightrightarrows \mathbb{C}]$ between the discrete categories based on the sets $\mathscr{M}$ and $\mathbb{C}$, to objects $\mathscr{O}_{f}$ in the functor category $[\Gamma(M, \mathscr{G}), \Gamma(M, \mathscr{G})]$. In particular, this would bridge the approach based on higher geometric quantization with deformation quantization, extending what was described in Section 2.5 for the associative case $H=0$ and in Section 2.6 in the case of singular magnetic charge distributions.

It is also an open issue as to whether or not the constructions discussed here, which deal with topologically trivial bundle gerbes, can be adapted to classes of nontrivial bundle gerbes, without the use of a trivializing open cover. For example, it would be interesting to extend the weak projective 2 -representations to compact Lie groups using the quantum field theory construction of three-cocycles in [35]. In this case the definition of the 2-Hilbert space is more involved due to technical difficulties related to the non-torsion gerbes [2]. However, at least the definition of a higher weak two-cocycle should carry over.

Acknowledgements. This article is based in part on work done in collaboration with Severin Bunk, Vladislav Kupriyanov, Dionysios Mylonas, Lukas Müller and Peter Schupp, who we warmly thank for many productive conversations. We thank Damien Calaque, Alberto Cattaneo and Jouko Mickelsson for helpful discussions and correspondence, and Branislav Jurčo, Christian Sämann, Urs Schreiber and Martin Wolf for the invitation to speak at the LMS/EPSRC/Durham Symposium on 'Higher Structures in M-Theory'. This work was supported by the COST Action MP1405 QSPACE, funded by the European Cooperation in Science and Technology (COST), and by the Consolidated Grant ST/P000363/1 from the UK Science and Technology Facilities Council (STFC).

Key words. Higher quantization, nonassociative geometry, magnetic monopoles, non-geometric strings, bundle gerbes

\section{References}

[1] C. Saemann and R. J. Szabo, Groupoids, loop spaces and quantization of 2-plectic manifolds, Rev. Math. Phys. 25 (2013) 1330005 [1211.0395 [hep-th] ].

[2] S. Bunk, C. Saemann, and R. J. Szabo, The 2-Hilbert space of a pre-quantum bundle gerbe, Rev. Math. Phys. 30 (2018) 1850001 [1608.08455 [math-ph]].

[3] S. Bunk and R. J. Szabo, Fluxes, bundle gerbes and 2-Hilbert spaces, Lett. Math. Phys. 107 (2017) 1877 [1612.01878 [hep-th]].

[4] M. Gunaydin and B. Zumino, Magnetic charge and non-associative algebras, in: "Old and New Problems in Fundamental Physics: Meeting in Honour of G.C. Wick," p.43-53, 1984.

[5] M. Grutzmann, $H$-twisted Lie algebroids, J. Geom. Phys. 61 (2011) 476 [1005.5680 [math.DG] ].

[6] C. Castelnovo, R. Moessner, and S. L. Sondhi, Magnetic monopoles in spin ice, Nature 451N7174 (2008) 42 [0710.5515 [cond-mat.str-e1]].

[7] D. J. P. Morris and others, Dirac strings and magnetic monopoles in spin ice Dy2Ti2O7, Science 326 (2009) 411 [1011.1174 [cond-mat.mtrl-sci]].

[8] R. J. Szabo, Magnetic monopoles and non-associative deformations of quantum theory, J. Phys. Conf. Ser. 965 (2018) 012041 [1709.10080 [hep-th]].

[9] R. Blumenhagen and E. Plauschinn, Non-associative gravity in string theory?, J. Phys. A 44 (2011) 015401 [1010.1263 [hep-th]].

[10] D. Luest, T-duality and closed string non-commutative (doubled) geometry, JHEP 1012 (2010) 084 [1010.1361 [hep-th]].

[11] R. Blumenhagen, A. Deser, D. Luest, E. Plauschinn, and F. Rennecke, Non-geometric fluxes, asymmetric 
strings and non-associative geometry, J. Phys. A 44 (2011) 385401 [1106.0316 [hep-th]].

[12] D. Mylonas, P. Schupp, and R. J. Szabo, Membrane sigma-models and quantization of non-geometric flux backgrounds, JHEP 1209 (2012) 012 [1207.0926 [hep-th]].

[13] L. Freidel, R. G. Leigh, and D. Minic, On the noncommutativity of closed string zero modes, Phys. Rev. D 96 (2017) 066003 [1707.00312 [hep-th]].

[14] R. Jackiw, 3-cocycle in mathematics and physics, Phys. Rev. Lett. 54 (1985) 159.

[15] R. Jackiw, Dirac's magnetic monopoles (again), Int. J. Mod. Phys. A 19S1 (2004) 137 [hep-th/0212058].

[16] S. Bunk, L. Mueller, and R. J. Szabo, Geometry and 2-Hilbert space for non-associative magnetic translations, 1804.08953 [hep-th].

[17] R. J. Szabo, Higher quantum geometry and nongeometric string theory, 1803.08861 [hep-th].

[18] T. T. Wu and C. N. Yang, Dirac monopole without strings: monopole harmonics, Nucl. Phys. B 107 (1976) 365.

[19] M. A. Soloviev, DiracâĂŹs magnetic monopole and the Kontsevich star product, J. Phys. A 51 (2018) 095205 [1708.05030 [math-ph]].

[20] M. Kontsevich, Deformation quantization of Poisson manifolds, I, Lett. Math. Phys. 66 (2003) 157 [q-alg/9709040].

[21] M. Kontsevich, Deformation quantization of algebraic varieties, Lett. Math. Phys. 56 (2006) 271 [math/0106006].

[22] P. Severa, Quantization of Poisson families and of twisted Poisson structures, Lett. Math. Phys. 63 (2003) 105 [math. QA/0205294].

[23] P. Aschieri, I. Bakovic, B. Jurco, and P. Schupp, Noncommutative gerbes and deformation quantization, hep-th/0206101.

[24] D. Mylonas, P. Schupp, and R. J. Szabo, Non-geometric fluxes, quasi-Hopf twist deformations and nonassociative quantum mechanics, J. Math. Phys. 55 (2014) 122301 [1312.1621 [hep-th]].

[25] I. Bakas and D. Luest, 3-cocycles, non-associative star-products and the magnetic paradigm of $R$-flux string vacua, JHEP 1401 (2014) 171 [1309.3172 [hep-th]].

[26] A. Weinstein, The local structure of Poisson manifolds, J. Diff. Geom. 18 (1983) 523.

[27] M. V. Karasev, Analogues of the objects of Lie group theory for non-linear Poisson brackets, Izvestiya: Mathematics 28 (1987) 497.

[28] A. Coste, P. Dazord, and A. Weinstein, Groupoïdes symplectiques, Publications du Département de mathématiques (Lyon) (1987) 1.

[29] A. S. Cattaneo and P. Xu, Integration of twisted Poisson structures, J. Geom. Phys. 49 (2004) 187 [math.SG/0302268].

[30] V. G. Kupriyanov and R. J. Szabo, Symplectic realization of electric charge in fields of monopole distributions, Phys. Rev. D 98 (2018) 045005 [1803.00405 [hep-th]].

[31] K. C. H. Mackenzie and P. Xu, Integration of Lie bialgebroids, Topology 39 (2000) 445 [dg-ga/9712012].

[32] V. G. Kupriyanov, Recurrence relations for symplectic realization of (quasi)-Poisson structures, 1805.12040 [math-ph].

[33] M. K. Murray, Bundle gerbes, J. Lond. Math. Soc. 54 (1996) 403 [dg-ga/9407015].

[34] K. Waldorf, More morphisms between bundle gerbes, Theor. Appl. Categor. 18 (2007) 240 [math. CT/0702652].

[35] J. Mickelsson, From gauge anomalies to gerbes and gerbal actions, Clay Math. Proc. 12 (2010) 211 [0812.1640 [math.DG]]. 